\title{
Establishment of the Elastic Property Database of Fe-base Alloys
}

\author{
Di Su, Yan-Lin He*, Ji-Qiong Liu, Xiao-Gang Lu
}

School of Materials Science and Engineering, Shanghai University, Shanghai, 200444, China

${ }^{*}$ Corresponding author. Tel.: +86 21 56331472. E-mail addresses: ylhe@staff.shu.edu.cn.

Keywords: Fe-base alloys, elastic properties, CALPHAD approach.

\begin{abstract}
The elastic properties, including bulk modulus and Young's modulus, for the bcc and fcc phases of Fe-base alloys have been studied at room temperature and atmospheric pressure. The temperature dependence of the elastic properties of the elemental metals, i.e. Fe, $\mathrm{Al} . .$. and ... has been modeled by using the CALPHAD approach and the first-principles calculations. Reasonable model parameters have been obtained giving a best description of most experimental data.
\end{abstract}

\section{Introduction}

Elastic properties are fundamental properties of solid materials. The knowledge of these properties is essential in numerous areas spanning from solid-state physics to materials selection in mechanical design. Elastic properties are also important in establishing the relationship between solid material deformation and stress. The elastic property databases act as an indispensable complement to the growing CALPHAD databases. Recently various CALPHAD models of molar volume have been reviewed by $\mathrm{He}$ et al.[1]. However, for Fe-base systems, modeling of elastic properties is still lacking.

For cubic structures, several relationships between single elastic constants, $C_{i j}$ and shear modulus(G), Young's modulus(E), bulk modulus(B) listed below have been proposed to calculate the elasticity of polycrystalline[2],so we can derive these elastic properties by using the single crystal elastic constants.

$$
\begin{aligned}
& B=\frac{1}{3}\left(C_{11}+2 C_{12}\right) \\
& G=\frac{3 C_{44}+C_{11}-C_{12}}{5} \\
& E=\frac{9 B G}{3 B+G}
\end{aligned}
$$

In the present work, we will study the elastic properties, B and E, of the Fe-X binary systems.

\section{Methodology}

\section{Experimental method for Young's modulus of pure metals}

The Young's modulus is usually measured by ultrasonic method at finite temperatures. The materials listed in Table 1 were cut to a sample of $60 \times 10 \times 1 \mathrm{~mm}$ and then solid-solution treated under vacuum in quartz capsules at certain temperatures for 48 hours followed by water quenching.

The experimental device is RFDA MF SYSTEM 21, which is based on the theory that any material has its own unique resonance frequency (in the range of $10-20000 \mathrm{~Hz}$ ), and with the relationship between time and amplitude in free attenuation process[4], the Young's modulus (E), shear modulus $(\mathrm{G})$ and Poisson's ratio (Poisson's ratio) can be determined.

Table 1 Raw materials

\begin{tabular}{ccc}
\hline Element & Purity & Treatment temperature/K \\
\hline Fe & $99.99 \%$ & 1000 \\
Mo & $99.9 \%$ & 1170 \\
$\mathrm{Al}$ & $99.999 \%$ & 700 \\
$\mathrm{Ta}$ & $99.9 \%$ & 1000 \\
\hline
\end{tabular}




\section{First-principles calculations}

Bulk modulus of pure elements and Fe-X binary system in bcc and fcc structure are used in the present work. These data can be evaluated from the experimental data as fitting model parameters. On the other hand, it is not difficult to obtain these values from the first-principles calculations. In the present work, for data which is hard to find in the literature we performed the first-principles calculations in order to make reliable prediction for Fe-X binary systems.

First-principles calculations, based on density functional theory, require only knowledge of the atomic species and crystal structure and yield the structural, elastic, electronic, magnetic and thermal properties related to the electronic structure and total energy of a given structure. In this article, the first-principles calculations were performed using the plane wave method with projector augmented wave (PAW) pseudo-potentials[4], as implemented in the Vienna ab initio simulation package (VASP)[5], The electron wave functions are expanded in a standard plane wave basis set with an energy cutoff of $400 \mathrm{eV}$.

\section{Elastic properties of pure elemental metals at finite temperatures}

In this work of assessing the temperature dependence of elastic properties of pure elements, we use a mathematic model:

$$
\varphi=\mathrm{a}+\mathrm{b} \times \mathrm{T}+\mathrm{c} \times \mathrm{T}^{2}
$$

where $\varphi$ represents the elastic properties $\mathrm{E}$ or $\mathrm{B}$, and $\mathrm{a}, \mathrm{b}$ and $\mathrm{c}$ are fitting parameters. The assessed parameters for pure elemental metals are listed in Table 2.

\begin{tabular}{lllll}
\multicolumn{5}{c}{ Table 2: Parameters for pure elemental metals } \\
\hline System & $\begin{array}{l}\text { Property } \\
\text { (E: Young's Modulus }\end{array}$ & $\mathrm{b}$ & $\mathrm{C}$ \\
& B: Bulk modulus) & & \\
\hline FCC Al & $\mathrm{E}$ & $7.028 \mathrm{E}+01$ & $2.114 \mathrm{E}-03$ & $-4.903 \mathrm{E}-05$ \\
& $\mathrm{~B}$ & $8.030 \mathrm{E}+01$ & $-1.319 \mathrm{E}-02$ & $-6.815 \mathrm{E}-06$ \\
FCC Cu & $\mathrm{E}$ & $1.397 \mathrm{E}+02$ & $-3.074 \mathrm{E}-02$ & $-2.220 \mathrm{E}-05$ \\
& $\mathrm{~B}$ & $1.427 \mathrm{E}+02$ & $-1.430 \mathrm{E}-02$ & $-1.036 \mathrm{E}-05$ \\
BCC Fe & $\mathrm{E}$ & $2.062 \mathrm{E}+02$ & $1.148 \mathrm{E}-02$ & $-1.162 \mathrm{E}-04$ \\
& $\mathrm{~B}$ & $1.735 \mathrm{E}+02$ & $-2.122 \mathrm{E}-02$ & $7.308 \mathrm{E}-06$ \\
FCC Fe & $\mathrm{B}$ & $2.150 \mathrm{E}+02$ & $-3.423 \mathrm{E}-02$ & $-9.977 \mathrm{E}-06$ \\
BCC Mo & $\mathrm{E}$ & $2.605 \mathrm{E}+02$ & $-2.777 \mathrm{E}-03$ & $-2.530 \mathrm{E}-05$ \\
& $\mathrm{~B}$ & $2.705 \mathrm{E}+02$ & $-5.262 \mathrm{E}-04$ & $-2.260 \mathrm{E}-05$ \\
FCC Ni & $\mathrm{E}$ & $2.420 \mathrm{E}+02$ & $-5.159 \mathrm{E}-02$ & $-2.150 \mathrm{E}-05$ \\
FCC Pb & $\mathrm{B}$ & $4.908 \mathrm{E}+01$ & $-1.382 \mathrm{E}-02$ & $-2.796 \mathrm{E}-06$ \\
FCC Pt & $\mathrm{E}$ & $1.832 \mathrm{E}+02$ & $-1.615 \mathrm{E}-02$ & $-1.016 \mathrm{E}-05$ \\
& $\mathrm{~B}$ & $2.901 \mathrm{E}+02$ & $-2.363 \mathrm{E}-02$ & $-4.856 \mathrm{E}-06$ \\
BCC Ta & $\mathrm{E}$ & $1.843 \mathrm{E}+02$ & $-1.080 \mathrm{E}-02$ & $-1.253 \mathrm{E}-05$ \\
& $\mathrm{~B}$ & $1.942 \mathrm{E}+02$ & $1.142 \mathrm{E}-02$ & $-6.497 \mathrm{E}-05$ \\
BCC W & $\mathrm{B}$ & $3.146 \mathrm{E}+02$ & $-1.246 \mathrm{E}-02$ & $-1.474 \mathrm{E}-05$ \\
\hline
\end{tabular}

Firstly we will discuss the bulk and Young's modulus of BCC Fe and FCC Fe.

\section{Temperature dependence of elastic properties of BCC Fe and FCC Fe}

The single crystal elastic constants $\mathrm{C}_{11}, \mathrm{C}_{12}$ and $\mathrm{C}_{44}$ can be used to calculate the temperature dependence of bulk modulus by using Eq.(1)[7-9]. In this work, selected values of the experimentally determined single crystal elastic constants[7] along with theoretical single crystal elastic constants predicted by electronic calculations[10-11] were used to optimize the temperature dependence of bulk modulus of BCC Fe, the temperature dependence of young's modulus of BCC Fe is assessed by using experiment data and Rayne and Chandrasekhar's work[7] by using Eq.(2) and Eq.(3). 


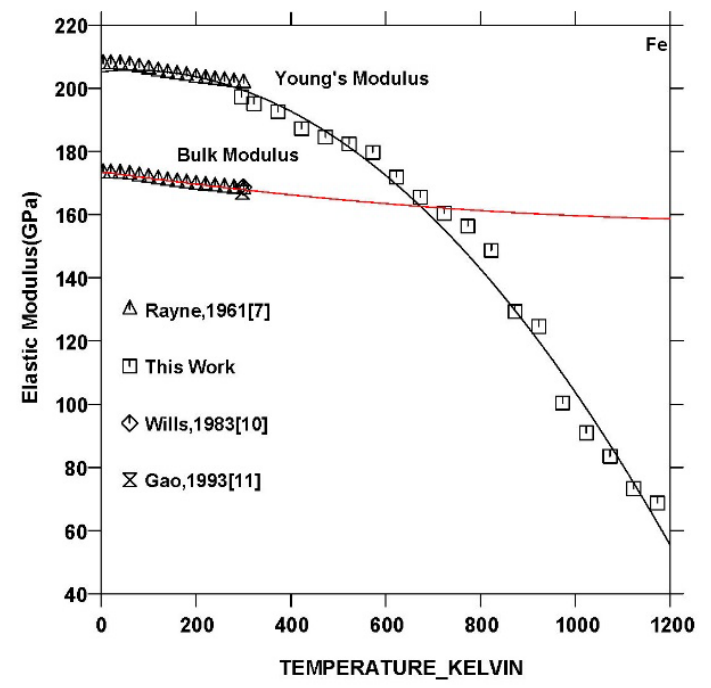

Fig.1. The temperature dependence of elastic properties of BCC Fe.

Fig. 1 shows the temperature dependence of young's modulus and bulk modulus of BCC Fe, as seen that the bulk modulus Wills and Harrison[10] calculated is higher than those derived from the single crystal data and data from Gao et al.[11] is lower. It also demonstrated that the experimental data of young's modulus has a good consistent with the values from Rayne and Chandrasekhar's work.

Unlike BCC Fe, the experimental elastic constant data of FCC Fe is very limited. Brandes and Brook[13] used an empirical correlation to predict the bulk modulus of FCC Fe under 298K. A selected value of the experimental single crystal elastic constants along with the bulk modulus value under room temperature is listed in Table 3.

Table 3: Selected elastic constants of FCC Fe

\begin{tabular}{llllll}
\hline Source & Temperature, $\mathrm{K}$ & $\mathrm{C}_{11}, 10^{10} \mathrm{~N} / \mathrm{m}^{2}$ & $\mathrm{C}_{12}, 10^{10} \mathrm{~N} / \mathrm{m}^{2}$ & $\mathrm{C}_{44}, 10^{10} \mathrm{~N} / \mathrm{m}^{2}$ & $\mathrm{~B}, 10^{10} \mathrm{~N} / \mathrm{m}^{2}$ \\
\hline$[11]$ & 1428 & 15.4 & 14.39 & 7.84 & 14.727 \\
{$[12]$} & 1428 & 18.1 & 15.60 & 8.33 & 16.433 \\
{$[13]$} & 298 & & & & 20.4 \\
\hline
\end{tabular}

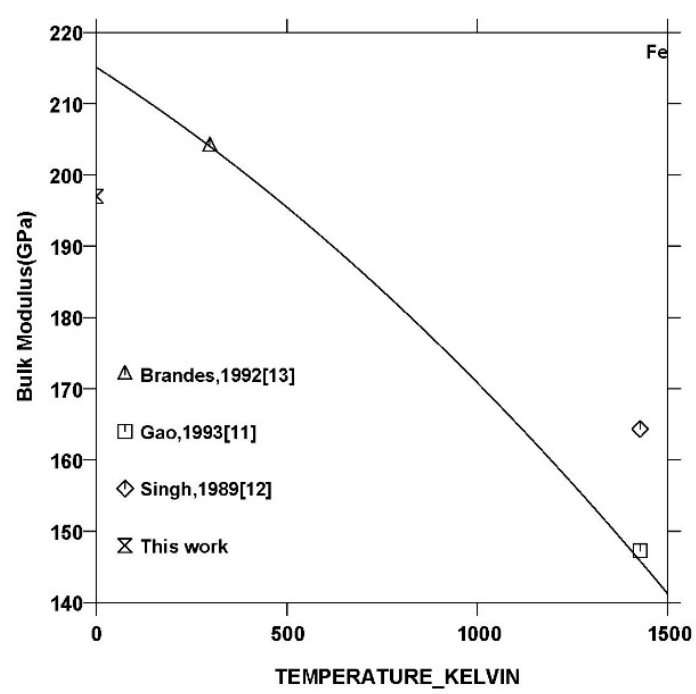

Fig.2. The temperature dependence of bulk modulus of FCC Fe.

As seen in Fig.2, bulk modulus for fcc Fe at 1428K derived from the data of Singh[12] is different from Gao et al.'s[11] by about 33\%. Although the assessment is empirical, it gives bulk modulus for fcc Fe at $0 \mathrm{~K}$ as $2.151 \times 10^{11} \mathrm{~Pa}$, which is not so different from $1.97 \times 10^{11} \mathrm{~Pa}$ calculated by the first-principles calculations.

In the following, we will present the temperature dependence of elastic properties of other 
related elements.

\section{Temperature dependence of elastic properties of other related elements}

\section{1) FCC Cu}

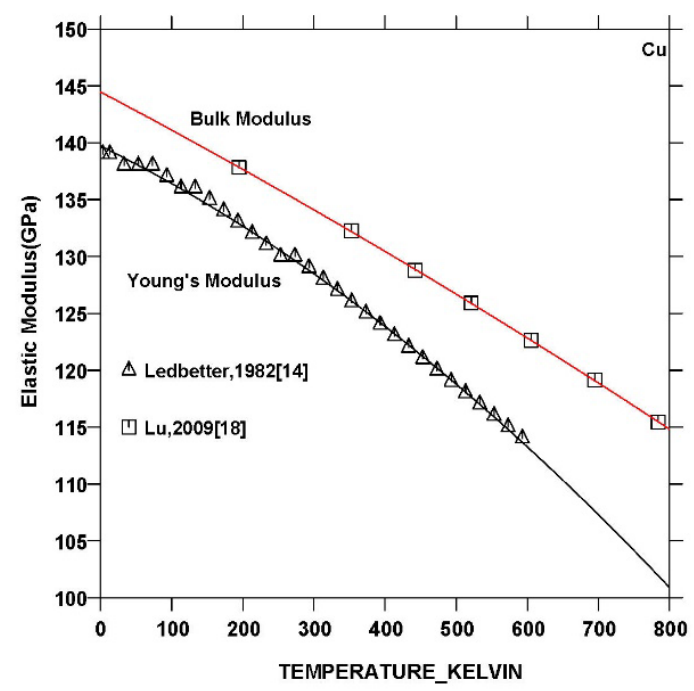

Fig.3. The temperature dependence of elastic properties of fcc $\mathrm{Cu}$

As seen in Fig.3, for Young's modulus of fcc $\mathrm{Cu}$, the value Ledbetter[14] calculated has already had a good agreement among various data sets[15-17], so we just use it to do the assessment. For the bulk modulus of fcc $\mathrm{Cu}$, the calculated isothermal bulk modulus by Lu and Chen[18] was used in this assessment.

2) FCC Al

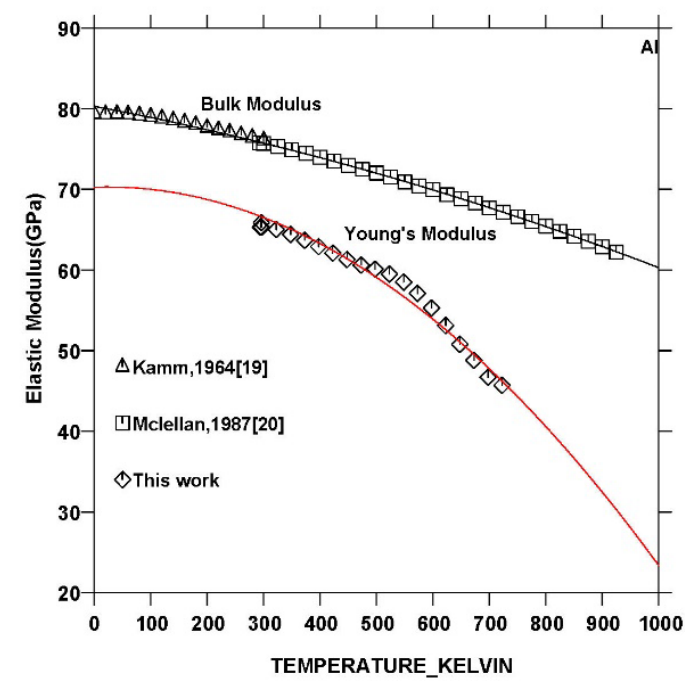

Fig.4. The temperature dependence of elastic properties of fcc $\mathrm{Al}$

The bulk modulus of fcc Al was derived from the single elastic constants measured by the ultrasonic echo method. As can be seen from Fig.4, the values from Kamm and Alers's work[19] and Mclellan and Ishikawa's[20] were successfully fitted in the present assessment.

For the temperature dependence of young's modulus of fcc $\mathrm{Al}$, we used $99.999 \% \mathrm{Al}$ to measure the young's modulus of fcc Al between $294.25 \mathrm{~K}$ and $722.65 \mathrm{~K}$. 


\section{3) BCC Mo}

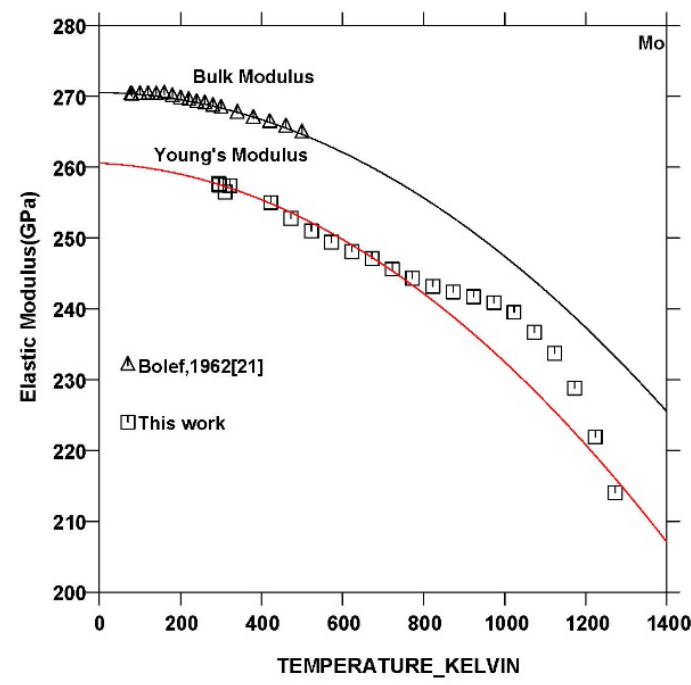

Fig.5. The temperature dependence of elastic properties of bcc Mo.

For the bulk modulus of bcc Mo, the value obtained by Featherson[22] is lower than other data[21] and was not used in the present assessment. As for the temperature dependence of young's modulus of bcc Mo, we used 99.9\% Mo to perform the experiments. As seen in Fig.5, the data within $800 \mathrm{~K}$ to $1200 \mathrm{~K}$ are about 5GPa higher than the assessment.

4) FCC Pb

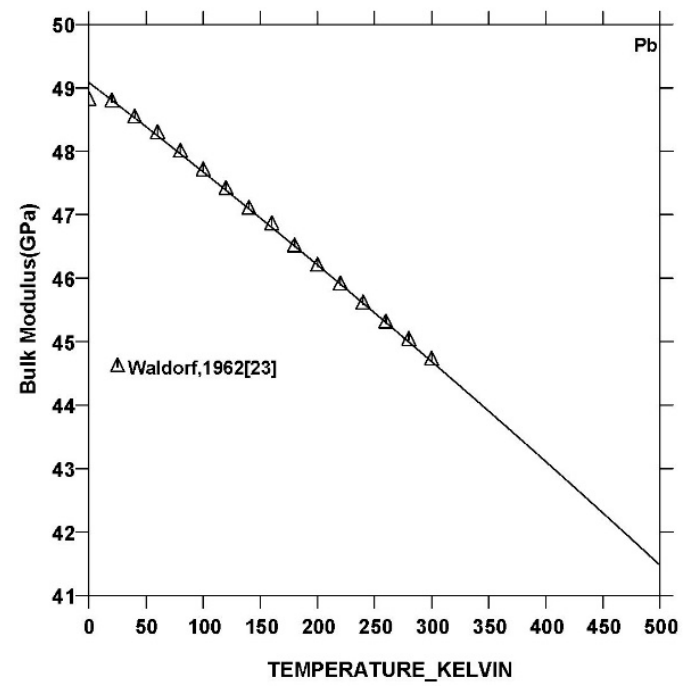

Fig.6. The temperature dependence of bulk modulus of fcc $\mathrm{Pb}$.

Waldorf[23] used the pulse-echo technique to measure the bulk modulus of fcc $\mathrm{Pb}$, as seen in Fig.6. 


\section{5) FCC Pt}

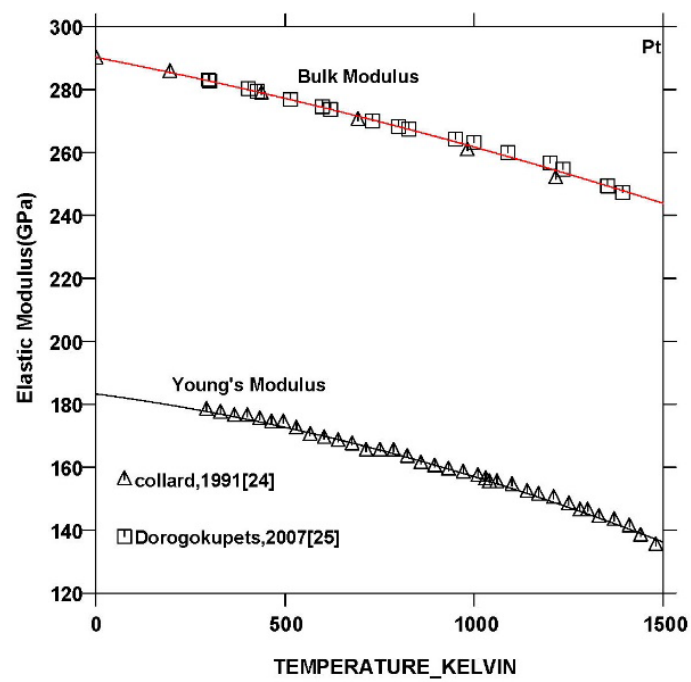

Fig.7. The temperature dependence of elastic properties of fcc Pt

Collard and Mclellan[24] used pulse-echo technique to measure the young's modulus of fcc Pt, as seen in Fig.7. For the bulk modulus of fcc Pt, the data from Collard and Mclellan[24] along with the measured values from Dorogokupets and Oganov[25] show a good agreement with the assessment.

6) BCC Ta

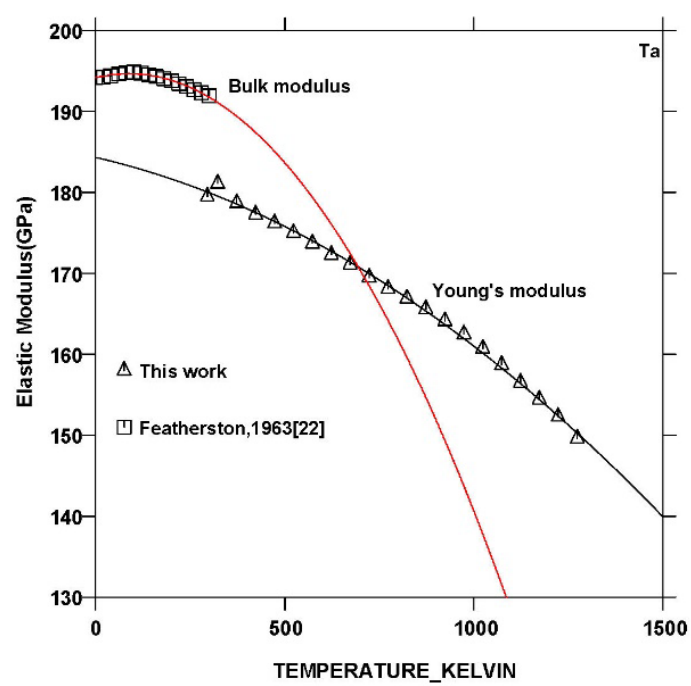

Fig.8. The temperature dependence of elastic properties of bcc Ta

We used 99.9\% Ta to measure the young's modulus of bcc Ta from $298 \mathrm{~K}$ to $1300 \mathrm{~K}$, as shown in Fig.8. The bulk modulus of bcc Ta was measured by Featherson and Neighbours[22] and adopted in the present assessment. 


\section{7) BCC W}

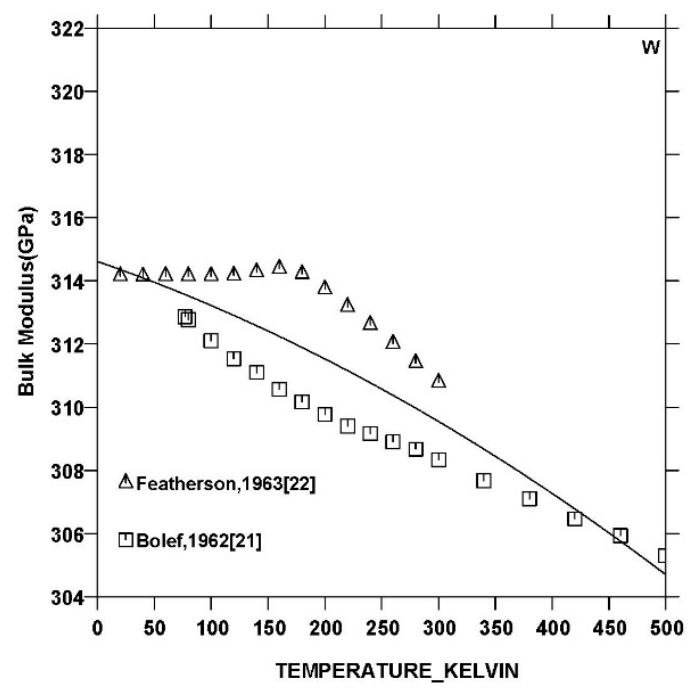

Fig.9. The temperature dependence of bulk modulus of bcc W

Both Bolef and Klerk[21] and Featherson and Neighours[22] used the ultrasonic pulse-echo technique to measure the elastic constants. Bulk modulus was derived from these data by using Eq.(1). As can be seen from Fig.9 that the difference between these two sets of data is about 3GPa, which is reasonable.

\section{8) FCC Ni}

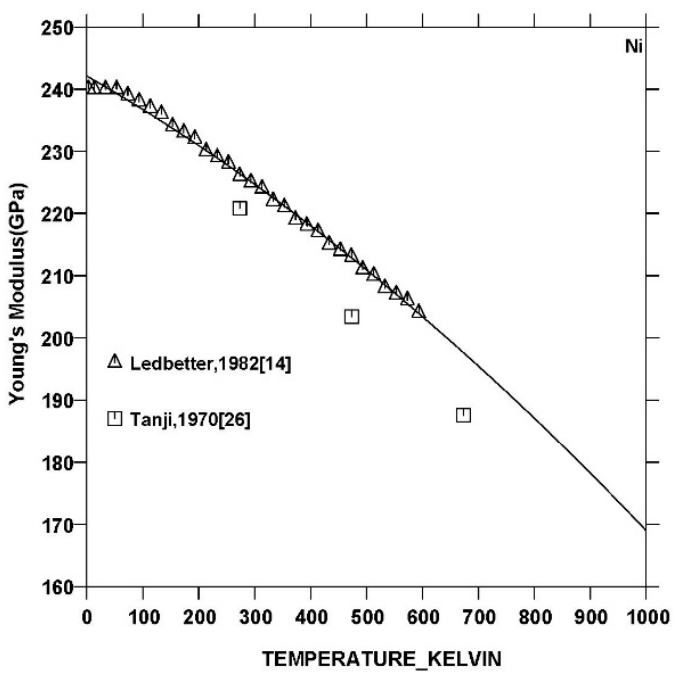

Fig.10. The temperature dependence of young's modulus of fcc Ni

For fcc Ni, the value Tanji[26] measured is lower than assessment by about 5GPa.

\section{General Discussion}

Above all, the temperature dependence of elastic properties of pure elements is successfully established. In the following we will discuss the composition dependence of elastic properties of $\mathrm{Fe}-\mathrm{X}$ at $298 \mathrm{~K}$ under atmosphere pressure. The elastic properties of pure elements at $298 \mathrm{~K}$ under atmosphere pressure are listed in Table 4.

Table 4: The elastic properties of pure elements at 298K under atmosphere pressure

\begin{tabular}{ccc}
\hline System & $\mathrm{E}, 10^{10} \mathrm{~N} / \mathrm{m}^{2}$ & $\mathrm{~B}, 10^{10} \mathrm{~N} / \mathrm{m}^{2}$ \\
\hline FCC Al & 7.571 & 6.53 \\
BCC Co & 20.18 & $17.70\left(^{*}\right)$ \\
BCC Cr & 22.17 & $12.74\left(^{*}\right)$ \\
FCC Cu & 12.86 & 13.74 \\
BCC Fe & 20.11 & 16.81 \\
FCC Fe & 22.83 & 20.4 \\
BCC Mo & 26.83 & 25.81 \\
\hline
\end{tabular}




\begin{tabular}{lcc}
\hline FCC Ni & 22.34 & $19.96\left(^{*}\right)$ \\
FCC Pb & 16.10 & 4.47 \\
FCC Pt & 17.82 & 28.21 \\
BCC Ta & 18.01 & 19.20 \\
BCC W & 40.84 & 30.94 \\
\hline
\end{tabular}

The value with* is calculated by the first-principles calculations

\section{Composition dependence of the Young's modulus of Fe-X}

Experiment shows that the addition of the other constituent elements will change the young's modulus of the material, thus change the elastic properties of the matrix material.

According to the above assessments of temperature dependence of the Young's modulus of pure elements and experimental data of Fe-X alloys, the prediction of composition dependence of the Young's modulus of Fe-X at 298K under atmosphere pressure has been made.

Most of the data came from G. R. Speich's[27] research, in which an ultrasonic pulse-echo technique was chosen to measure the shear modulus and young's modulus of some alloys, then the bulk modulus and Poisson's ratio can be derived from E and G by Eq.(4) and Eq.(5) for isotropic bodies.

$$
\begin{aligned}
& B=\frac{E G}{3(3 G-E)} \\
& \mu=\frac{E}{2 G}-1
\end{aligned}
$$

The values for BCC Fe at 298.15K are $\mathrm{E}=199.314 \mathrm{GPa}, \mathrm{B}=167.90 \mathrm{GPa}$ from the assessment from the temperature dependence of young's modulus and bulk modulus of BCC Fe. The values for FCC Fe are $\mathrm{B}=204 \mathrm{GPa}$ from the assessment of the temperature dependence of bulk modulus of FCC Fe. Ghosh and Olson[2] predicted the temperature dependence of shear modulus for fcc Fe. With bulk modulus and shear modulus of fcc Fe, we can derive young's modulus of fcc Fe as E $=228.3 \mathrm{GPa}$ using Eq. (4).

In the assessments below, the young's modulus of pure elements at $298 \mathrm{~K}$ under atmosphere pressure are fixed, we use the experimental data and values calculated by first-principle calculation to optimize by CALPHAD methods.

\section{FCC Fe-Ni system}

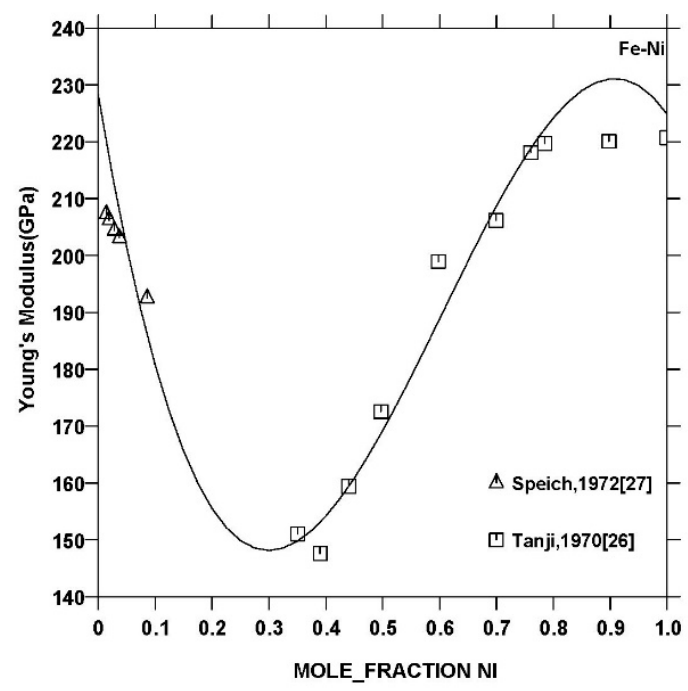

Fig.11. The composition dependence of young's modulus of fcc Fe-Ni at 298K.

For fcc Fe-Ni system, Tanji[26] used electrostatic driving method to measure the fundamental flexural and the second torsional resonance frequencies of fcc Fe-Ni specimens, and Young's modulus and shear modulus were determined. As seen in Fig.11, the assessed composition dependence of young's modulus of fcc Fe-Ni agrees with the data measured by Tanji[26] and Speich[27] except the data in the composition range between 0.8 to 1 . 


\section{BCC Fe-Co system}

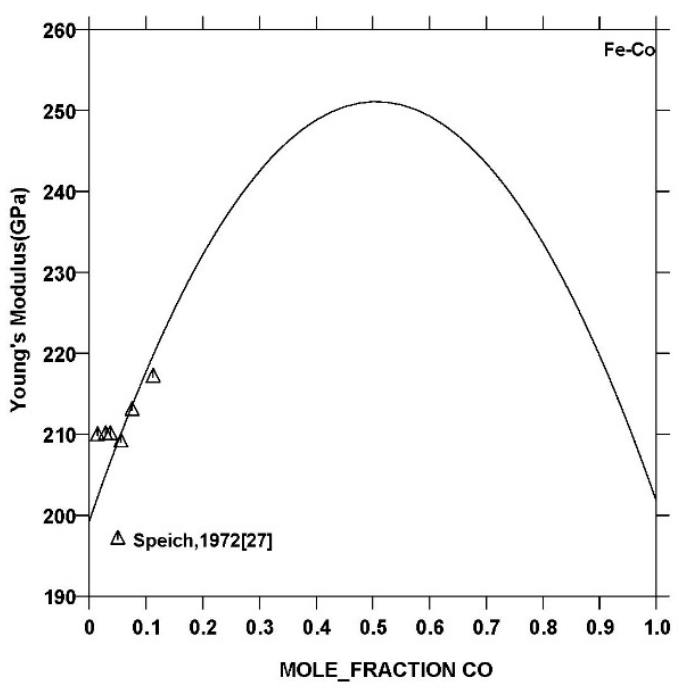

Fig.12. The composition dependence of young's modulus of bcc Fe-Co at 298K

For bcc Fe-Co system, Gump et al.[28] measured the bulk modulus and elastic constants of bcc Co, we can derive young's modulus for bcc Co to be E=201.78GPa. As seen in Fig.12, it presents a parabolic trend, i.e. the young's modulus of bcc Fe-Co increases sharply during 0 to 0.6, then drops until the mole fraction of Co reaches 1.

BCC Fe-Cr system

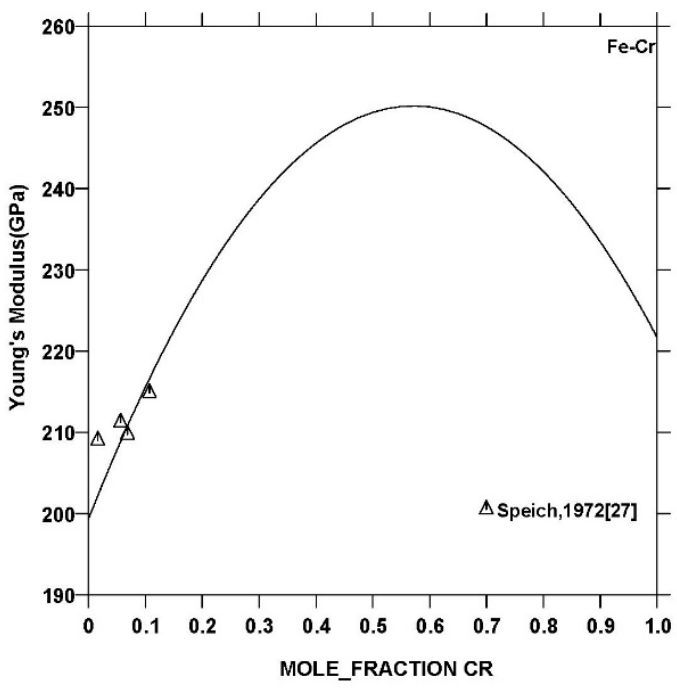

Fig.13. The composition dependence of young’s modulus of bcc Fe-Cr at 298K

For bcc Fe-Cr system, we calculate $\mathrm{B}=127.37 \mathrm{GPa}$ at $0 \mathrm{~K}$ by the first-principles calculations, and because the Poisson's ratio remains constant between $0 \mathrm{~K}$ and 298K, we derived the Young's modulus of bcc Fe-Cr as E=221.68GPa using Eq. (4) and Eq. (5). The Young's modulus at 298K under atmosphere pressure is shown in Fig.13. 


\section{FCC Fe-Pt system}

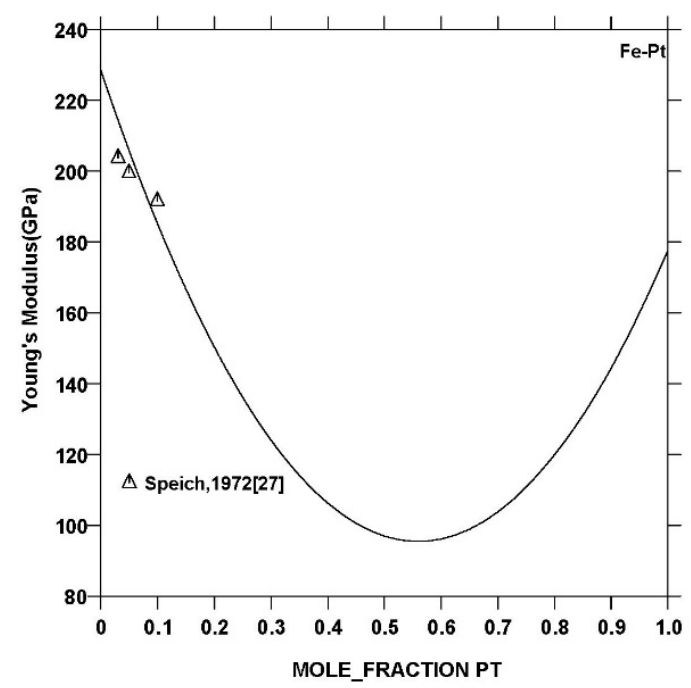

Fig.14. The composition dependence of young's modulus of fcc Fe-Pt at 298K

For fcc Fe-Pt system, as seen in Fig.14, the agreement between 0 and 0.2 is acceptable.

\section{General Discussion}

In this work of assessing the composition dependence of young's modulus of Fe-X system, we used interaction parameter, $\mathrm{L}_{0}$ and $\mathrm{L}_{1}$, to optimize the young's modulus of Fe-X systems by CALPHAD method. The agreement with the experiments is acceptable. Due to lacking of the data of Fe-X binary system, we can't establish all related Fe-X system assessments. The interaction parameters for these Fe-X systems are listed in Table 5.

Table 5: Interaction parameters for Young's modulus of Fe-X systems

\begin{tabular}{llll}
\hline System & & $\mathrm{L}_{0}$ & $\mathrm{~L}_{1}$ \\
\hline BCC Fe-Co & $\mathrm{E}_{\mathrm{Fe}-\mathrm{Co}}^{\mathrm{bcc}}$ & $2.021 \mathrm{E}+02$ & \\
BCC Fe-Cr & $\mathrm{E}_{\mathrm{Fe}-\mathrm{Cr}}^{\mathrm{bcc}}$ & $1.555 \mathrm{E}+02$ & $-3.711 \mathrm{E}+02$ \\
FCC Fe-Ni & $\mathrm{E}_{\mathrm{Fe}-\mathrm{Ni}}^{\mathrm{cc}}$ & $-2.293 \mathrm{E}+02$ & \\
FCC Fe-Pt & $\mathrm{E}_{\mathrm{Fe}-\mathrm{Pt}}^{\mathrm{fcc}}$ & $-4.240 \mathrm{E}+02$ & \\
\hline
\end{tabular}

\section{Conclusion}

Knowledge of elastic properties is important in materials selection and materials design. In the present work, we proposed to establish the elastic properties database of $\mathrm{Fe}-\mathrm{X}$ alloys by using the CALPHAD method. After systematically assessing the elastic properties of pure elements which related with Fe-X system, we optimize the composition dependence of young's modulus of Fe-X binary system at room temperature and atmospheric pressure.

\section{Acknowledgements}

The financial support from the National Key Project of Science and Technology (Grant number: 20 12ZX06004-012) is gratefully acknowledged.

\section{Reference}

[1] Y.-L. He, X.-G. Lu, N.-Q. Zhu, B. Sundman, Chin. Sci. Bull. (Chin. Ver) 58 (2013) 3642-3646.

[2] Ghosh G, Olson G B. The isotropic shear modulus of multi-component Fe-base solid solutions[J]. Acta materialia, 2002, 50(10): 2655-2675.

[3] Shimada M, Matsushita K, Kuratani S, et al. Temperature dependence of Young's modulus and internal friction in alumina, silicon nitride, and partially stabilized zirconia ceramics[J]. Journal of the American Ceramic Society, 1984, 67(2): C-23C-24. 
[4] Tomioka Y, Yuki N. Bend stiffness of copper and copper alloy foils[J]. Journal of materials processing technology, 2004, 146(2): 228-233.

[5] Kresse G, Joubert D. From ultrasoft pseudopotentials to the projector augmented-wave method[J]. Physical Review B, 1999, 59(3): 1758.

[6] Kresse G, Furthmüller J. Efficient iterative schemes for ab initio total-energy calculations using a plane-wave basis set[J]. Physical Review B, 1996, 54(16): 11169.

[7] Rayne JE, Chandrasekhar BS. Physic Review 1961;122:1714.

[8] Leese J, Lord Jr AE. J Appl Phys 1968;39:3986.

[9] Dever DJ. J Appl Phys 1972;43:3293.

[10]Wills JM, Harrison WA. Physic Review B 1983;28:4363.

[11]Gao F, Johnston RL, Murrell JN. J Phys Chem 1993;97:12073.

[12] Singh N. Physic State Solid Basic 1989;156:K33.

[13]Brandes EA, Brook GB, editors. Smithels metals reference handbook. Oxford: Butterworth-Heinemann; 1992.

[14]H. M. Ledbetter. Temperature behavior of Young's moduli of forty engineering alloys. Cryogenics; 1882.

[15]Chang Y A, Himmel L. Temperature dependence of the elastic constants of $\mathrm{Cu}$, Ag, and $\mathrm{Au}$ above room temperature[J]. Journal of Applied Physics, 1966, 37(9): 3567-3572.

[16]Biswas S N, Van't Klooster P, Trappeniers N J. Effect of pressure on the elastic constants of noble metals from -196 to $+25^{\circ} \mathrm{C}$ and up to 2500 bar: II. Silver and gold[J]. Physica B+ C, 1981, 103(2): 235-246.

[17] Overton Jr W C, Gaffney J. Temperature variation of the elastic constants of cubic elements. I. Copper[J]. Physical Review, 1955, 98(4): 969.

[18]Lu X G, Chen Q. A CALPHAD Helmholtz energy approach to calculate thermodynamic and thermophysical properties of fcc Cu[J]. Philosophical Magazine, 2009, 89(25): 2167-2194.

[19]Kamm G N, Alers G A. Low-Temperature Elastic Moduli of Aluminum[J]. Journal of Applied Physics, 1964, 35(2): 327-330.

[20]McLellan R B, Ishikawa T. The elastic properties of aluminum at high temperatures[J]. Journal of Physics and Chemistry of Solids, 1987, 48(7): 603-606.

[21]Bolef D I, De Klerk J. Elastic Constants of Single - Crystal Mo and W between $77^{\circ}$ and $500^{\circ}$ K[J]. Journal of applied Physics, 1962, 33(7): 2311-2314.

[22]Featherston F H, Neighbours J R. Elastic constants of tantalum, tungsten, and molybdenum[J]. Physical Review, 1963, 130(4): 1324.

[23] Waldorf D L. Low-temperature elastic moduli of lead[J]. Journal of Applied Physics, 1962.

[24]Collard S M, McLellan R B. High-temperature elastic constants of platinum single crystals[J]. Acta metallurgica et materialia, 1992, 40(4): 699-702.

[25]Dorogokupets P I, Oganov A R. Ruby, metals, and MgO as alternative pressure scales: A semiempirical description of shock-wave, ultrasonic, x-ray, and thermochemical data at high temperatures and pressures[J]. Physical Review B, 2007, 75(2): 024115.

[26] Tanji Y, Shirakawa Y, Moriya H. Young's Modulus, Shear Modulus and Compressibility of Fe-Ni (fcc) Alloys[J]. 1970.

[27]Speich G R, Schwoeble A J, Leslie W C. Elastic constants of binary iron-base alloys[J]. Metallurgical Transactions, 1972, 3(8): 2031-2037.

[28]Gump J, Xia H, Chirita M, et al. Elastic constants of face-centered-cubic cobalt[J]. Journal of applied physics, 1999, 86(11): 6005-6009. 